Check for updates

Cite this: RSC Adv., 2017, 7, 43531

Received 13th July 2017

Accepted 4th September 2017

DOI: 10.1039/c7ra07712b

rsc.li/rsc-advances

\section{Origin of the optical anisotropy and the electronic structure of Ru-based double perovskite oxides: DFT and XPS studies $\uparrow$}

\author{
Anup Pradhan Sakhya, (D) *a D. P. Rai, ${ }^{\text {b }}$ Md. Sariful Sheikh, ${ }^{a}$ Manabendra Mukherjee, ${ }^{c}$ \\ Alo Dutta ${ }^{d}$ and T. P. Sinha ${ }^{a}$
}

\begin{abstract}
Experimental and theoretical studies of the electronic structure of $\operatorname{Pr}_{2} \mathrm{LiRuO}_{6}(\mathrm{PLR}), \mathrm{Nd}_{2} \mathrm{LiRuO}_{6}$ (NLR) and $\mathrm{Sm}_{2} \mathrm{LiRuO}_{6}$ (SLR) crystals are reported. The linear optical properties of these compounds have been investigated using density functional theory calculations. The optical anisotropy in these compounds is revealed from their computed optical properties, such as their complex dielectric functions and refractive indices. The calculations show that SLR has a relatively large birefringence $(\Delta n=0.06)$ compared to NLR and PLR, which is important for mid-infrared nonlinear optical applications. We propose that the $\mathrm{O} 2 \mathrm{p}$ to $\mathrm{Ru} 4 \mathrm{~d}$ transition is primarily responsible for the origin of optical activity in these materials while the origin of the optical anisotropy in these materials results from the asymmetrically oriented $\mathrm{Ru}-\mathrm{O}$ bonds in the $\mathrm{RuO}_{6}$ octahedra of the unit cell. The evolution of the Ru 3d core-level signals as obtained from the $\mathrm{X}$-ray photoemission spectroscopy measurements provide confirmation of a dynamic increment in the electron correlations as we move from PLR and NLR to SLR.
\end{abstract}

\section{Introduction}

The structural and the physical properties of double perovskite oxides with the general formula $\mathrm{A}_{2} \mathrm{BB}^{\prime} \mathrm{O}_{6}$ and distinct transition metal ions at the $\mathrm{B}$ and $\mathrm{B}^{\prime}$ sites have been an active area of research because of the diverse physical properties and structural chemistry exhibited by this family of oxides. ${ }^{1}$ Recently, the electronic properties of mixed-metal oxides containing ruthenium, particularly those which adopt the perovskite structure, have gained tremendous interest. This interest has been stimulated due to developments in a number of fields such as colossal magnetoresistance ${ }^{2,3}$ and superconductivity. ${ }^{4}$ Complex oxides with ruthenium show diverse properties, for example, rutile type $\mathrm{RuO}_{2}$ is a normal metal and the density of states (DOS) obtained from band structure calculations is in good agreement with its photoemission spectra. ${ }^{5} \mathrm{Bi}_{2} \mathrm{Ru}_{2} \mathrm{O}_{7}$ is a Pauliparamagnetic metal whereas pyrochlore-type $\mathrm{Y}_{2} \mathrm{Ru}_{2} \mathrm{O}_{7}$ is an insulator and exhibits a local-moment behaviour. ${ }^{6}$ Further, $\mathrm{Sr}_{2} \mathrm{RuO}_{4}$ has attracted interest due to its unusual electronic and magnetic properties such as non-s-wave superconductivity, ${ }^{7}$

\footnotetext{
${ }^{a}$ Department of Physics, Bose Institute, 93/1 Acharya Prafulla Chandra Road, Kolkata 700009, India. E-mail: npshakya31@gmail.com

${ }^{b}$ Department of Physics, Pachhunga University College, Aizawl 796001, Mizoram, India 'Saha Institute of Nuclear Physics, 1/AF, Bidhan Nagar, Kolkata 700064, India ${ }^{d}$ Department of Condensed Matter Physics and Material Sciences, S. N. Bose National Centre for Basic Sciences, Block-JD, Sector-III, Salt Lake, Kolkata-700098, India

$\dagger$ Electronic supplementary information (ESI) available. See DOI: 10.1039/c7ra07712b
}

orbital ordering in $\mathrm{La}_{4} \mathrm{Ru}_{2} \mathrm{O}_{10}$ (ref. 8) and non-Fermi-liquid behaviour in $\mathrm{La}_{4} \mathrm{Ru}_{6} \mathrm{O}_{19} \cdot{ }^{9} \mathrm{SrRuO}_{3}$ is a rare example of a $4 \mathrm{~d}$ ferromagnet $\left(T_{\mathrm{c}} \approx 165 \mathrm{~K}\right) .^{10}$ This change in the electronic behaviour has been attributed to the change in the electronelectron correlation as suggested by Kim et al. ${ }^{11}$ The conclusion has been made based on the fits of the experimental core level X-ray photoemission (XP) spectra. Recently, Makowski et al. have reported the structural and magnetic properties of the $\mathrm{Ln}_{2} \mathrm{LiRuO}_{6}$ double perovskites where $\mathrm{Ln}=\mathrm{Pr}, \mathrm{Nd}, \mathrm{Sm}, \mathrm{Eu}, \mathrm{Gd}$, $\mathrm{Tb}$ using synchrotron X-ray, neutron powder diffraction, and magnetic susceptibility measurements for the first time. ${ }^{12}$ Beyond this, not much is known about these materials. The motivation behind this paper is twofold. Firstly, we want to study the linear optical properties of these materials and see if these materials are birefringent. Birefringent materials are very important and interesting in optical communications and laser industry as they can modulate the polarization of light and are vital crystalline materials in producing optical devices such as optical isolators, circulators, polarization beam splitters and photo storage devices. ${ }^{13-17}$ The birefringence value of a material can be obtained by measuring the refractive index by using the minimum deviation method. However, large size crystals of good optical quality are needed which is very difficult to synthesize. Hence density functional theory (DFT) calculations play a very important role to predict the birefringence value which is very significant in choosing optical materials with suitable birefringence. ${ }^{17,18}$ Additionally, birefringence is necessary for phase matching conditions during the optical second harmonic experiments. ${ }^{19}$ Secondly, we have made an attempt to 
understand the electronic structure of these materials using XP spectroscopy and DFT calculations. A detailed theoretical understanding of the intrinsic physical properties of these materials will be very significant not only for fundamental research but also for technological applications.

\section{Experiment}

$\mathrm{A}_{2} \mathrm{LiRuO}_{6}(\mathrm{~A}=\mathrm{Pr}, \mathrm{Nd}, \mathrm{Sm})$ compounds have been synthesized by the solid-state reaction method. For the synthesis of $\mathrm{A}_{2} \mathrm{LiRuO}_{6}$, the reagent grades of $\mathrm{Pr}_{2} \mathrm{O}_{3}$ (Sigma Aldrich), $\mathrm{Sm}_{2} \mathrm{O}_{3}$ (Alfa Aesar), $\mathrm{Nd}_{2} \mathrm{O}_{3}$ (Alfa Aesar) (prefired at $1000{ }^{\circ} \mathrm{C}$ for $12 \mathrm{~h}$ ), $\mathrm{RuO}_{2}$ (Alfa Aesar) (pre-fired at $450{ }^{\circ} \mathrm{C}$ for $12 \mathrm{~h}$ ) and $20 \mathrm{~mol} \%$ excess of $\mathrm{Li}_{2} \mathrm{CO}_{3}$ (Loba Chemie) (pre-fired at $80{ }^{\circ} \mathrm{C}$ for $12 \mathrm{~h}$ ) are taken in stoichiometric ratio and mixed in the acetone medium. The resultant mixture is then dried and calcined at $980{ }^{\circ} \mathrm{C}$ for $12 \mathrm{~h}$ and brought to room temperature by cooling at the rate of $100{ }^{\circ} \mathrm{C} \mathrm{h}^{-1}$. The material is then mixed again in the acetone medium with an additional $20 \mathrm{~mol} \%$ excess of $\mathrm{Li}_{2} \mathrm{CO}_{3}$ and then finally heated at $980{ }^{\circ} \mathrm{C}$ for $12 \mathrm{~h}$ (cooled at the rate of $100{ }^{\circ} \mathrm{C} \mathrm{h}^{-1}$ ). The excess $\mathrm{Li}_{2} \mathrm{CO}_{3}$ has been added because of the volatilization of $\mathrm{Li}$ and is essential for the preparation of pure samples. ${ }^{12}$ The calcined powders are pelletized into discs $(8 \mathrm{~mm}$ diameter and $1.5 \mathrm{~mm}$ thickness) using polyvinyl alcohol as a binder. These discs are then sintered at $1080{ }^{\circ} \mathrm{C}$ for $10 \mathrm{~h}$ and cooled to room temperature at the rate of $1{ }^{\circ} \mathrm{C} \mathrm{min}^{-1}$. The X-ray diffraction (XRD) pattern of the synthesized samples have been taken at the room temperature using Rigaku Miniflex-II X-ray powder diffractometer in the $2 \theta$ range from $10^{\circ}$ to $80^{\circ}$ by scanning at $0.02^{\circ}$ per step. The Rietveld refinement of the XRD pattern has been carried out using FULLPROF ${ }^{20}$ method. In the refinement process, the background is fitted with sixth order polynomial, while the peak shapes are described by pseudo-Voigt profiles. During the refinement, scale factor, zero correction, background, half width parameters, lattice parameters, positional coordinates and isotropic thermal parameters $\left(B_{\text {iso }}\right)$ are varied. No correlation between the positional and the thermal parameters are observed during refinement and as such, it is possible to refine all the parameters together. XP core-level spectra are taken with an Omicron Multiprobe (Omicron Nanotechnology GmbH., UK) spectrometer fitted with an EA125 hemispherical analyzer. A monochromated $1486.7 \mathrm{eV}$ X-ray source operated at $150 \mathrm{~W}$ has been used for the experiments. The analyzer pass energy is kept fixed at $40 \mathrm{eV}$ for all the scans. As the samples are insulating in nature, a low energy electron gun (SL 1000, Omicron) with a large spot size has been used to neutralize the samples. The potential of the electron gun is kept fixed at $-3 \mathrm{eV}$ for all the samples with respect to the ground. The binding energy (BE) has been determined with reference to the $\mathrm{C}$ 1s line at $284.8 \mathrm{eV}$.

\section{Computational details}

In this study, two computational methods within the framework of density functional theory (DFT) have been used in a complementary manner. The structural optimization and dielectric tensor are obtained by using the projector augmented wave
$(\mathrm{PAW})^{\mathbf{2 1 , 2 2}}$ method as implemented in the VASP. ${ }^{23}$ The electronic structure and the optical properties have been calculated using WIEN $2 \mathrm{k}$ code. ${ }^{24}$ For more details on computational parameters see Section $\mathrm{S} 1$ in ESI. $\dagger$

\section{Results and discussion}

\subsection{Structural study}

The stability of $\mathrm{A}_{2} \mathrm{LiRuO}_{6}$ to a first approximation is determined by the ratio of $\mathrm{A}-\mathrm{O}, \mathrm{Li}-\mathrm{O}, \mathrm{Ru}-\mathrm{O}$ bond lengths, which can be expressed as the tolerance factor given by

$$
T_{\mathrm{f}}=\frac{R_{\mathrm{A}}+R_{\mathrm{O}}}{\sqrt{2}\left(\frac{R_{\mathrm{Li}}+R_{\mathrm{Ru}}}{2}+R_{\mathrm{O}}\right)}
$$

where $R_{\mathrm{A}}, R_{\mathrm{O}}, R_{\mathrm{Li}}$, and $R_{\mathrm{Ru}}$ denote the ionic radii of $\mathrm{A}, \mathrm{O}, \mathrm{Li}$ and $\mathrm{Ru}$ ions respectively. The tolerance factor calculated using the ionic radius of $\operatorname{Pr}(1.179 \AA)$, Nd (1.27 $\AA$ ), $\operatorname{Sm}(1.24 \AA), \operatorname{Li}(0.76 \AA)$, $\mathrm{Ru}(0.62 \AA)$ and $\mathrm{O}(1.4 \AA)$ are found to be $0.87,0.90$ and 0.89 for PLR, NLR and SLR respectively. Usually a cubic perovskite structure is obtained for $T_{\mathrm{f}}$ close to unity, whereas it can be lower symmetrical structure (orthorhombic, monoclinic) for lower value of tolerance factor. This value of the tolerance factor suggests that the room temperature crystal structure of $\mathrm{A}_{2} \mathrm{LiRuO}_{6}$ should have a lower symmetry than cubic structure. The distortion from the ideal cubic double perovskite structure is a result of the tilting of the $\mathrm{LiO}_{6}$ and $\mathrm{RuO}_{6}$ octahedra, while maintaining their corner-sharing connectivity. This is commonly observed for compositions that include an A-cation which is too small to fill the 12 -fold coordination site and would result in reduced eight-fold coordination environment. To verify the same, we have performed the Rietveld refinement of the room-temperature XRD patterns of $\mathrm{A}_{2} \mathrm{LiRuO}_{6}$ using the Fullprof software. ${ }^{20}$ The XRD patterns of $\mathrm{A}_{2} \mathrm{LiRuO}_{6}$ are shown in Fig. 1. A good agreement between the observed (symbols) and refined (lines) data suggests that the compounds are crystallized in the monoclinic perovskite structure with space group $P 2_{1} / n$. As both the A-O and ( $\left.\mathrm{Li}, \mathrm{Ru}\right)-\mathrm{O}$ bond lengths are limited to a narrow range; for a double perovskite structure, the only way to accommodate smaller A-cations is by distorting the $\mathrm{Li}-$ $\mathrm{O}-\mathrm{Ru}$ bond angles. This feature of the rotated octahedron, common among all $P 2_{1} / n$ ordered double perovskites, requires that the $(\mathrm{Li}, \mathrm{Ru})-\mathrm{O}_{6}$ octahedrons have different tilt angles as a result of the differences in the size and the distortion of the two polyhedron. Consequently, the $\beta$ angle of a monoclinic unit cell can be viewed as a measure of the structural distortion. The lattice parameters, atomic positions, reliability factors, bond lengths and bond angles for the different constituent ions are listed in (Table S4 and Table S5 respectively in ESI $\dagger$ ). In these materials $\mathrm{Pr}$, Nd and $\mathrm{Sm}$ have co-ordination number 8 instead of 12. The co-ordination number of these cations has been reduced because some of the anions move too far away from the A-cation for lower symmetry double perovskite oxides (DPOs) due to the octahedral tilting which causes the first coordination sphere about the A cation to change. It has been proposed by Woodward $^{25}$ that any anion more than $3.00 \AA$ away from the Acation of DPOs may be considered to be outside the 


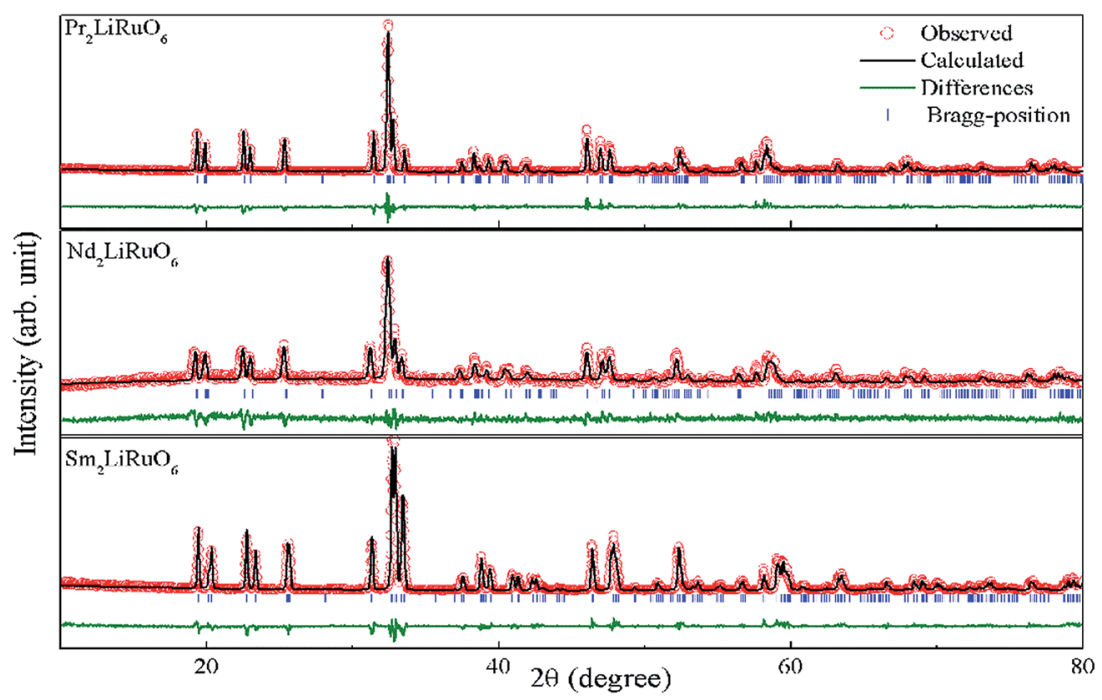

Fig. 1 Rietveld refinement plot of $\mathrm{A}_{2} \mathrm{LiRuO}_{6}$ at room temperature. The experimental points are represented by circles and the solid line represents the simulated XRD data. The vertical marks in the middle show the positions calculated for Bragg reflection. The trace at the bottom (green line) is the plot of the difference between the observed and the calculated pattern.

coordination sphere. A schematic presentation of the $\mathrm{A}_{2} \mathrm{LiRuO}_{6}$ cell is shown in Fig. S1 in ESI, $\dagger$ with the distribution of ions at crystallographic positions $4 \mathrm{e}$ for $\mathrm{A}$ ions, $2 \mathrm{~d}$ for $\mathrm{Li}$ ions, $2 \mathrm{c}$ for $\mathrm{Ru}$ ions and $4 \mathrm{e}$ for $\mathrm{O}$ ions as given in Table S4 in ESI.† Each Li and $\mathrm{Ru}$ ions are surrounded by six $\mathrm{O}$ ions, constituting $\mathrm{LiO}_{6}$ and $\mathrm{RuO}_{6}$ octahedra, respectively.

\subsection{Spin polarized electronic band structure and density of states}

The spin-polarized band structures of $\mathrm{A}_{2} \mathrm{LiRuO}_{6}(\mathrm{~A}=\mathrm{Pr}, \mathrm{Nd}, \mathrm{Sm})$ compounds along the high-symmetry directions in the Brillouin zone are shown in Fig. 2, where similarities in the energy bands are evident. For the clarity of the figures, the energy bands in the region from $-6 \mathrm{eV}$ to $6 \mathrm{eV}$ are shown in Fig. 2(a)-(c). In these calculations, the zero of the energy scale is taken at the top of the valence band (VB). It is clear that the spin-up/down channels of PLR possesses an indirect energy band gap as the valence band maximum (VBM) is located at the $\Gamma$ symmetry point of the Brillouin zone (BZ) for spin up/down, while the conduction band minimum (CBM) is situated at the $R$ symmetry point for spin up ( $\uparrow)$ and at the $\Gamma$ point for spin down $(\downarrow)$. NLR also possesses an indirect energy band gap as the VBM is located at the $\Gamma$ symmetry point for spin up $(\uparrow)$ and $\Sigma$ symmetry point for spin down $(\downarrow)$, while the CBM is located at the $\Sigma$ symmetry point for spin up $(\uparrow)$ and $\Gamma$ symmetry point for spin down $(\downarrow)$. SLR however shows a direct band gap as the VBM is located at the $\Gamma$ symmetry point of the Brillouin zone (BZ) for both spin up $(\uparrow)$ /down $(\downarrow)$ whereas the CBM is situated at the $\Gamma$ symmetry point of the Brillouin zone (BZ) for both spin up $(\uparrow) /$ down $(\downarrow)$. The spin-polarized electronic band structure of $\mathrm{A}_{2} \mathrm{LiRuO}_{6}(\mathrm{~A}=\mathrm{Pr}, \mathrm{Nd}, \mathrm{Sm})$ reveals that all the compounds are insulators. The calculated band gaps are $3.1 \mathrm{eV}(\uparrow)$ and $1.6 \mathrm{eV}$ $(\downarrow)$ for PLR, $3.12 \mathrm{eV}(\uparrow)$ and $1.7 \mathrm{eV}(\downarrow)$ for NLR and $2.2 \mathrm{eV}(\uparrow)$ and $1.8 \mathrm{eV}(\downarrow)$ for SLR. It is observed that the energy gap of spin down $(\downarrow)$ is noticeably lower than that of spin up $(\uparrow)$. This is attributed to the fact that the location of Ru d states at the lower part of the conduction band (CB) for the spin down $(\downarrow)$ pushes the CBM towards the Fermi level $\left(E_{\mathrm{F}}\right)$ resulting in the reduced energy gap value for all these compounds. However, for the spin up ( $\uparrow)$, the Ru d states as well as A f states are shifted towards higher energies resulting in the increased spin up $(\uparrow)$ band gap.

The calculated DOS for the investigated compounds are shown in Fig. 3(a)-(c), which are helpful in identifying the character of band states at different energy levels. In the case of PLR, in the up-spin channel (shown by $\uparrow$ in [Fig. 3(a)]) the VB and the $\mathrm{CB}$ is mainly dominated by $\operatorname{Pr} \mathrm{f}$ and $\mathrm{Ru} \mathrm{d}$ states hybridized with $\mathrm{O} p$ and Li p states. The existence of Pr $\mathrm{f}$ states in the upper VB and lower CB have a significant effect on the energy band gap dispersion. The Ru d states lie near the $E_{\mathrm{F}}$ in the energy range from $-1.4 \mathrm{eV}$ to $0 \mathrm{eV}$ and in the lower part of the $\mathrm{VB}$ from $-4.5 \mathrm{eV}$ to $-5.1 \mathrm{eV}$, while the remaining states of $\mathrm{VB}$ are dominated by $\mathrm{O} \mathrm{p}$ character. In the down spin channel (shown by $\downarrow$ in [Fig. 3(a)]), the VB is mainly dominated by O $\mathrm{p}$ and Li p orbitals. Since the valency of Pr ion in PLR compound is $+3\left([\mathrm{Xe}] 4 \mathrm{f}^{2}\right)$, its energy states in the spin up channel are partially filled up and lies in the VB region whereas the energy states of Pr ion in the down spin channel (shown by $\downarrow$ in [Fig. 3(a)]), are completely empty and lies in the CB region. In the case of NLR, from the partial density of states (PDOS) plots, one can see that there exists a strong hybridization between the $\mathrm{Ru} d$ states and the $\mathrm{O} p$ states in the up-spin channel $(\uparrow)$ from $-1 \mathrm{eV}$ up to $E_{\mathrm{F}}$. In the VB the contribution of $\mathrm{Nd} 4 \mathrm{f}$ states lies in between the energy range from $-1.3 \mathrm{eV}$ to $-2.9 \mathrm{eV}$ hybridized with the $\mathrm{O}$ p states. The $\mathrm{CB}$ in the up-spin channel is mainly composed of $\mathrm{Ru} d$ states and $\mathrm{Nd} \mathrm{f}$ states. In the down spin channel (shown by $(\downarrow)$ in [Fig. 3(b)]), the VB is mainly composed of $\mathrm{O} p$ and Li s states. The band extending from 4 to $5.9 \mathrm{eV}$ in the $\mathrm{CB}$ is mainly occupied by the anti-bonding states of $\mathrm{Nd} 4 \mathrm{f}$. There is no contribution of $\mathrm{Nd} 4 \mathrm{f}$ states in the down spin channel, 

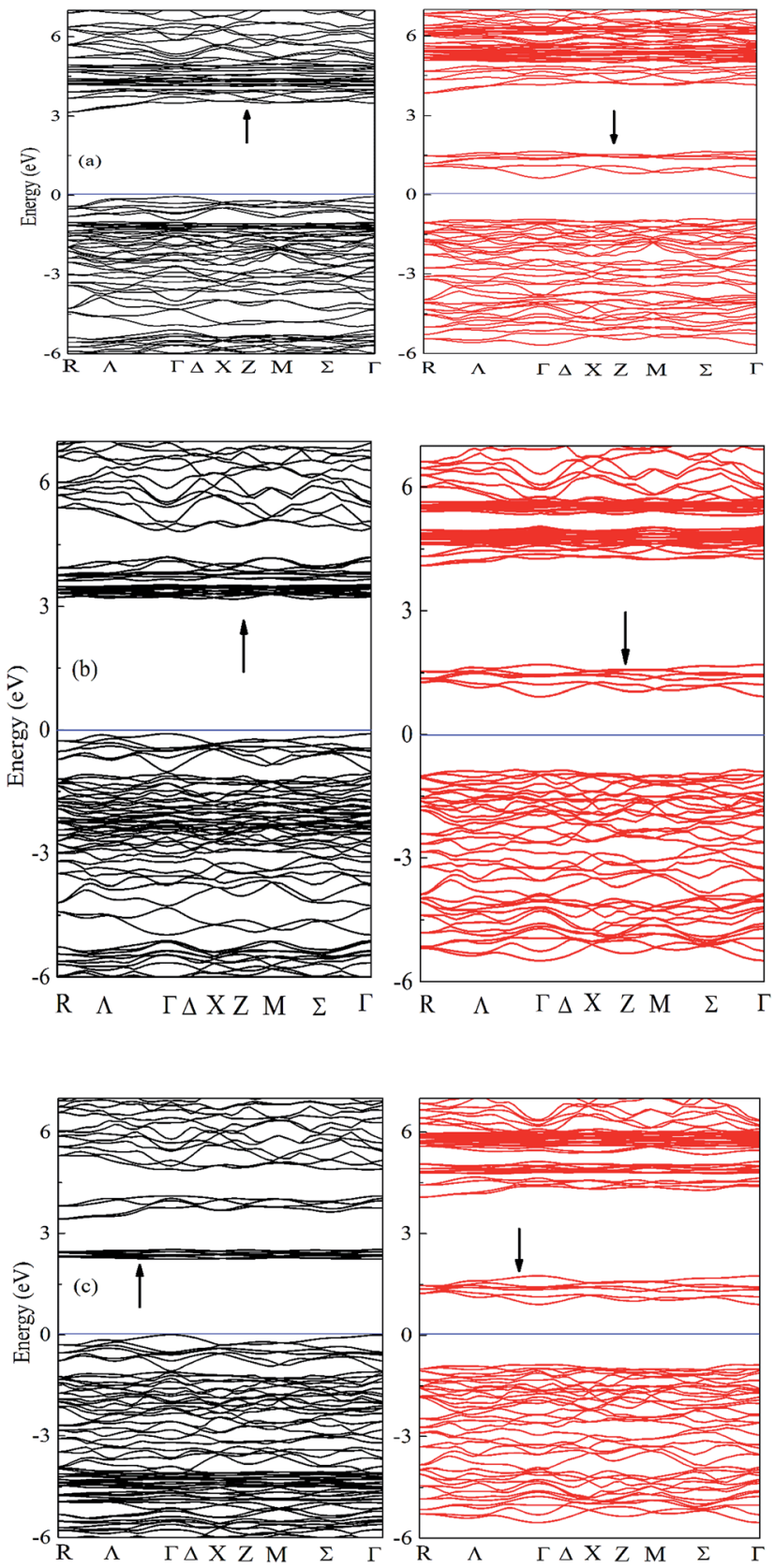

Fig. 2 Band structure of (a) PLR (b) NLR and (c) SLR within GGA + U. Bands in black (red) indicate the spin up (down) channel.

which is in conformity with the electronic configuration of $\mathrm{Nd}^{3+}$ $\left([\mathrm{Xe}] 4 \mathrm{f}^{3}\right)$ in NLR. The spin up channel of $\mathrm{Nd}(\uparrow)$ is not completely filled up, so some part of Nd states lies in the VB while the spin down channel is completely empty and lies in the CB. For SLR, in the up-spin channel (shown by $(\uparrow)$ in [Fig. 3(c)]), the VB consists mainly of Sm f, Ru d and O p orbitals. The states near the $E_{\mathrm{F}}$ have contributions mainly from $\mathrm{Ru} \mathrm{d}$ states hybridized with $\mathrm{O} p$ states. The $\mathrm{Sm} \mathrm{f}$ states lie in the energy region from $-3.8 \mathrm{eV}$ to $-5 \mathrm{eV}$ and are strongly localized. The $\mathrm{CB}$ in the up-spin channel is mainly composed of the anti-bonding states of Ru d and Sm $\mathrm{f}$ in the energy region from $3.3 \mathrm{eV}$ to $4.2 \mathrm{eV}$
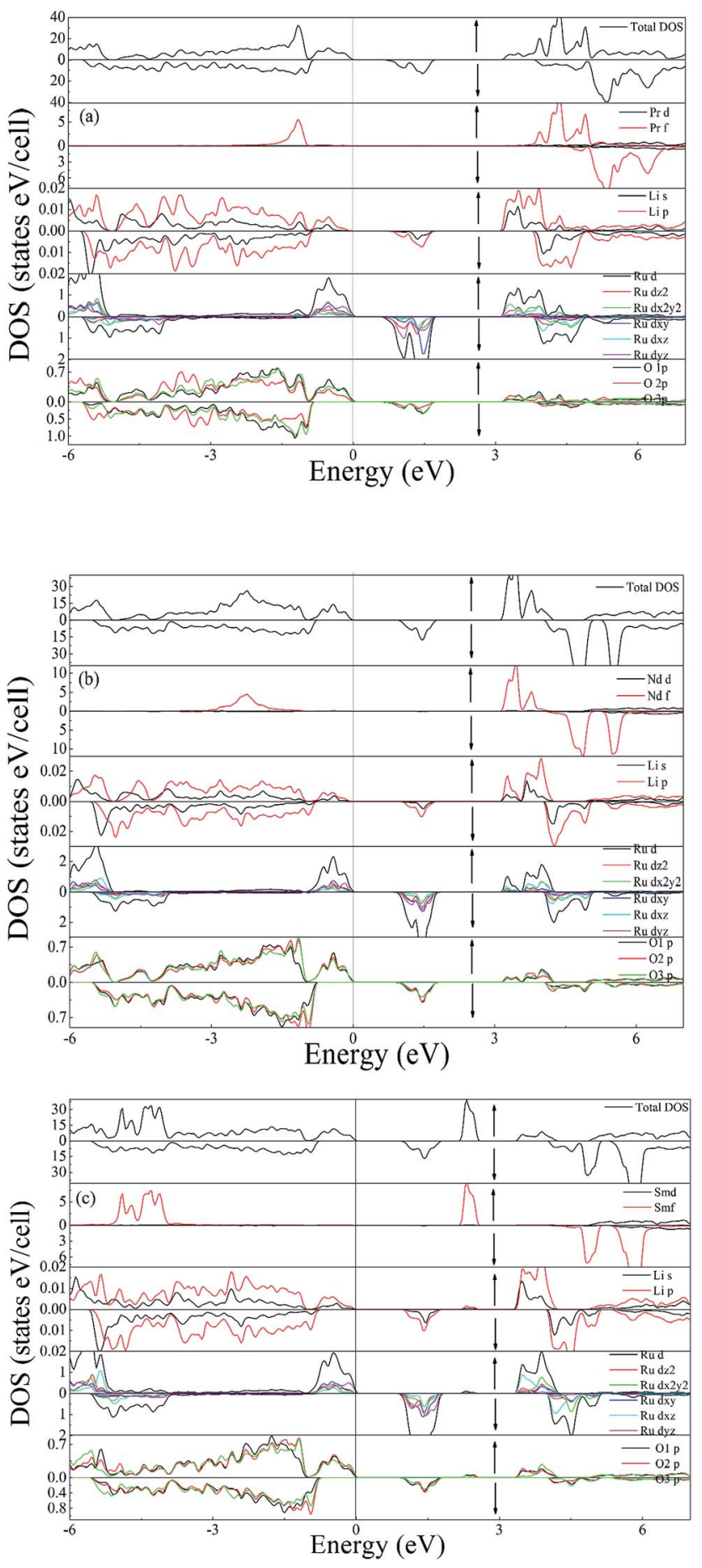

Fig. 3 Total DOS and Partial DOS of (a) PLR (b) NLR and (c) SLR using GGA + U.

and 2.1 to $2.6 \mathrm{eV}$ respectively. The VB in the down spin channel (shown by $(\downarrow)$ in [Fig. 3(c)]), is mainly composed of O p and Li p states. Since the valency of Sm ion in SLR is $+3\left([\mathrm{Xe}] 4 \mathrm{f}^{5}\right)$, it contains five electrons in the spin up channel so the major part of the Sm states lies in the VB while the spin down channel is completely empty and lies in the CB. 


\subsection{Linear optical properties}

The investigated compound crystallizes in the monoclinic phase with $P 2_{1} / n$ space group. This symmetry group has two dominant components of the dielectric tensor. These dielectric functions are $\varepsilon_{2}{ }^{\|}(\omega)$ and $\varepsilon_{2}{ }^{\perp}(\omega)$, corresponding to the electric field direction parallel and perpendicular to the crystallographic $c$ axis respectively (see Section S2 in ESI $\dagger$ ). The calculated dispersive part $\varepsilon_{1}(\omega)$ and the absorptive part $\varepsilon_{2}(\omega)$ of the complex dielectric function $\varepsilon(\omega)$ of $\mathrm{A}_{2} \mathrm{LiRuO}_{6}$ as a function of the photon energy are shown in Fig. 4(a)-(f). The imaginary part of the dielectric function illustrates the optical transition mechanism. The structures observed in the optical spectra originate from the transitions between the occupied and the unoccupied electronic energy states. The solid red and black lines in Fig. 4 represent the result along the $\varepsilon^{\perp}(\omega)$ and $\varepsilon^{\|}(\omega)$ polarization direction respectively. It can be seen from Fig. 4(b), (d) and (f) that the threshold energy for $\varepsilon_{2}(\omega)$ occurs at around 1.6, 1.7 and $1.8 \mathrm{eV}$ for peak A of PLR, NLR and SLR respectively. This point is $\Gamma_{\mathrm{v}}-\Gamma_{\mathrm{c}}$ splitting which gives the threshold for optical transitions between the highest VB and the lowest CB. This is known as the fundamental absorption edge. Beyond these points, the curve increases rapidly. This is because of the fact that the number of transitions from the VB to the $\mathrm{CB}$ which contributes toward $\varepsilon_{2}(\omega)$ curve increases rapidly. The peaks appearing in the imaginary part of the dielectric function are labelled as A, B and C. It should be stated that for the interpretation of a structure (peak) in the optical spectra of the systems, it does not seem realistic to give a single transition assignment resembling the energy of the peak since numerous transitions between the occupied and the unoccupied energy bands for a particular structure can be satisfied. In the case of PLR, the peak A mainly corresponds to the transitions from Ru d states lying at the top of the VB to the Pr f states in the CB. The peak B arises due to the transitions between the middle region of $\mathrm{O} 2 \mathrm{p}$ in the $\mathrm{VB}$ and $\mathrm{Ru}$ $\mathrm{d}$ states in the $\mathrm{CB}$. The peak $\mathrm{C}$ originates due to the transitions from the semi-core Ru d states to Pr f states as well as from the lowermost O 2p states in the VB to the Ru d states in the CB. For SLR, the transitions from the topmost Ru d states to $\mathrm{Sm}$ f states are responsible for the peak A whereas the peak B corresponds to the transitions from the $\mathrm{O} 2 \mathrm{p}$ states lying in the middle of the VB to $\mathrm{Ru} d$ states in the CB. The peak $\mathrm{C}$ arises due to the transitions from $\mathrm{Ru} d$ states lying at the semi-core region to $\mathrm{Sm} \mathrm{f}$ states in the $\mathrm{CB}$ and also for the transitions between the $\mathrm{O} 2 \mathrm{p}$ states at the lowermost VB to Ru d states. Similarly, for NLR, the peak A may be due to the transitions from $\mathrm{Ru} d$ to $\mathrm{Nd} f$, the peak $\mathrm{B}$ due to the transitions from $\mathrm{O} 2 \mathrm{p}$ to $\mathrm{Ru} \mathrm{d}$ states and the peak $\mathrm{C}$ due to the transitions from semi-core $\mathrm{Ru} d$ states to $\mathrm{Nd}$ f states (may also be due to the $\mathrm{O} 2 \mathrm{p}$ in the lowermost VB to Ru d states). It is clear from Fig. 4 that there is a considerable anisotropy between $\varepsilon_{1}{ }^{\perp}(\omega)$ and $\varepsilon_{1}{ }^{\|}(\omega)$ for $\mathrm{A}_{2} \mathrm{LiRuO}_{6}$ compounds [see Fig. 4(a), (c) and (e)]. In the low-frequency limit $(\omega \rightarrow 0)$; the magnitude of the dielectric response $\varepsilon_{1}(0)$ corresponds to the static optical dielectric constant $\left(\varepsilon_{\mathrm{s}}\right)$. The calculated static values of the dielectric function $\varepsilon_{1}{ }^{\perp}(0)$ and $\varepsilon_{1}{ }^{\|}(0)$ for these compounds are given in Table S6 in ESI. $\dagger$ The obtained values indicate a considerable anisotropy ${ }^{26}$ of the dielectric function for these
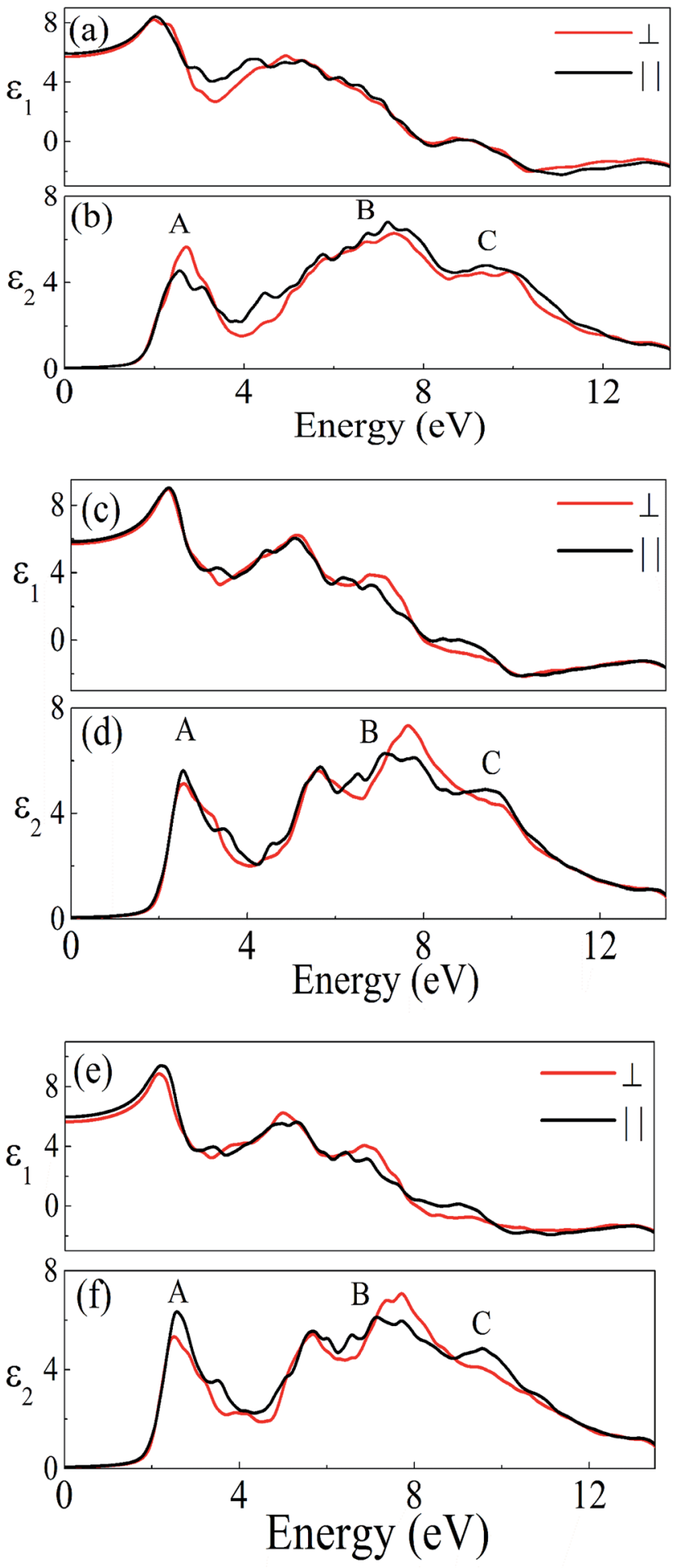

Fig. 4 Real $\varepsilon_{1}(\omega)$ and imaginary $\varepsilon_{2}(\omega)$ parts of the complex dielectric constant of ( $a$ and b) PLR (c and d) NLR and (e and f) SLR.

compounds. The uniaxial anisotropy is calculated using the following expression

$$
\left[\delta \varepsilon=\left(\varepsilon_{0}^{\|}-\varepsilon_{0}^{\perp}\right) / \varepsilon_{0}^{\text {total }}\right]
$$

and is found to be $0.02,0.01$ and 0.03 for PLR, NLR and SLR respectively indicating a considerable anisotropy ${ }^{26}$ of the 
dielectric function in these compounds. The optical dielectric constant is obtained from the average value of the $\varepsilon_{1}(0)$ resolved along the crystal axes and is given in Table S6 in ESI. $\uparrow$ The refractive index of these compounds along the different polarization directions have been calculated and is shown in Fig. S2, $\dagger$ which indicates the optically anisotropic nature of these compounds in the low energy range. For lower energies, refractive index values are almost constant and as the energy increases they attain a maximum value (near the absorption edge) and exhibit a decreasing tendency for higher energy values. We have also calculated the birefringence of these compounds which is the difference between the extraordinary and the ordinary refraction indices, $\Delta n(\omega)=n^{\|}(\omega)-n^{\perp}(\omega)$, where $n^{\|}(\omega)$ is the index of refraction for an electric field oriented along the $c$-axis and $n^{\perp}(\omega)$ is the index of refraction for an electric field perpendicular to the $c$-axis. Fig. 5 shows the birefringence $\Delta n(\omega)$ dispersion for $\mathrm{A}_{2} \mathrm{LiRuO}_{6}$ compounds. It is clear that the birefringence is important only in the nonabsorbing spectral range, which is below the energy gap. As we notice from Fig. $\mathbf{S} 2, \uparrow$ these compounds show a considerable anisotropy between $n^{\perp}(\omega)$ and $n^{\|}(\omega)$, as a consequence, they possess a large birefringence $\Delta n(\omega)$. The birefringence can be calculated from the linear response functions from which the anisotropy of the index of refraction is determined. We have found that these crystals possess a positive birefringence at zero energy equal to about $0.05,0.03$ and 0.06 for PLR, NLR, and SLR respectively. The birefringence is an important parameter to fulfil the phase-matching condition. ${ }^{27}$ Since the birefringence of SLR is large to obtain a phase matching for second harmonic generation, hence it can be used as a frequency doubling crystal. ${ }^{28}$ From the above analysis, it is clear that the calculated linear optical properties show a considerable anisotropy between the components along the polarization directions [100] and [001]. The strong hybridization of the $\mathrm{Ru} 4 \mathrm{~d}$ orbitals with the $\mathrm{O} 2 \mathrm{p}$ states indicates the relatively strong covalent bonds between $\mathrm{Ru}$ and $\mathrm{O}$. It is clear that the $\mathrm{RuO}_{6}$ octahedra has a big influence on the electronic structure and hence on the optical properties of these materials. Thus, we propose that the $\mathrm{RuO}_{6}$ octahedral groups are the main contributors to the optical anisotropy. We further propose that the major optical transitions would involve $\mathrm{Ru} 4 \mathrm{~d}$ states and $\mathrm{O} 2 \mathrm{p}$ states. In $\mathrm{A}_{2} \mathrm{LiRuO}_{6}$

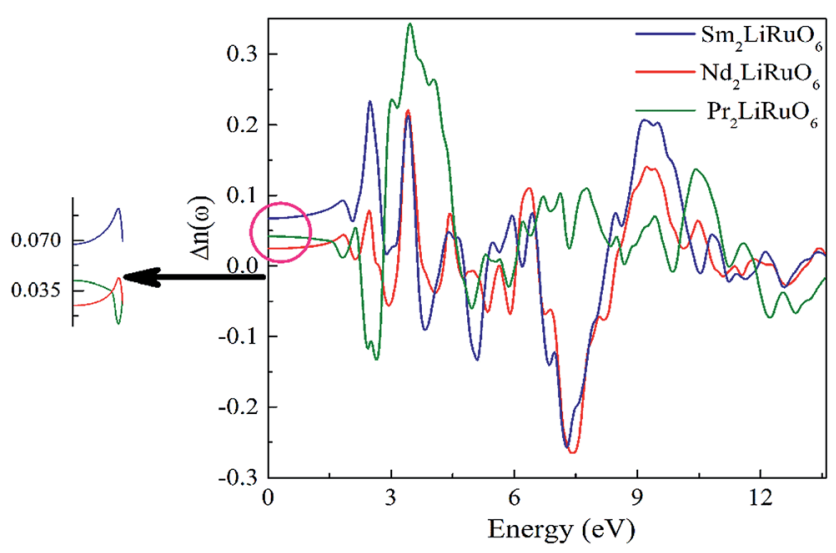

Fig. 5 The calculated birefringence $\Delta n(\omega)$. compounds, the density of $\mathrm{RuO}_{6}$ octahedra is different in the crystallographic $c$-direction with respect to the a and $b$ directions. Such anisotropic structural feature has been reported to result in anisotropic physical properties such as electrical conductivity. ${ }^{29}$ Thus, it is plausible that the orientation differences among the $\mathrm{RuO}_{6}$ octahedra lead to the different transition behaviour from $\mathrm{O} 2 \mathrm{p}$ to $\mathrm{Ru} 4 \mathrm{~d}$ states, which is the primary factor responsible for the optical activity of $\mathrm{A}_{2} \mathrm{LiRuO}_{6}$ compounds, rendering anisotropic optical behaviour. ${ }^{30}$

\subsection{X-ray photoemission spectroscopy study}

Fig. 6 shows the XP spectrum of $\mathrm{A}_{2} \mathrm{LiRuO}_{6}$ in the energy window of 0 to $1200 \mathrm{eV}$. The profile of the XP spectra are identified and indexed. The curve fitting of $\mathrm{A} 3 \mathrm{~d}$ and $\mathrm{Ru} 3 \mathrm{~d}$ core level peaks are performed to isolate the contribution of the various species to the total signal envelope. The high-resolution core-level spectrum of Ru 3d is shown in Fig. 7. The XP data for each spectrum has been normalized (zero-clipping) and plotted. The Ru 3d spectrum is complex and could be best fitted using a function with three sets of doublets which have been assigned as screened, unscreened and multiplet peaks. ${ }^{31}$ The six components are necessary to obtain a fit of good quality. These features have been observed in many other ruthenates and shown to be associated with different final state effects in photoemission process arising due to the screening of core hole by valence electrons..$^{32-35}$ Thus, we have fitted the experimental spectrum with three pairs of spin orbit peaks using XPSPEAK software. ${ }^{36}$ The Shirley method ${ }^{37}$ is used to subtract the background and then the experimental spectra are numerically fitted using the sum of the Lorentzian-Gaussian model. A typical peak fit performed on the compounds is depicted in Fig. 7.

The fitting parameters are given in Table 1. The spin orbit separation between the two Ru 3d peaks as shown in Table 1 corresponds well to the expected value of $4.17 \mathrm{eV}^{38}$ It is observed that the full-width at half-maximum (FWHM) of the $\mathrm{Ru} 3 \mathrm{~d}_{3 / 2}$ components is always larger than the $\mathrm{Ru} 3 \mathrm{~d}_{5 / 2}$ components. The comparison between $\mathrm{Ru} 3 \mathrm{~d}$ spectra in these materials reveals that the degree of localized character for $\mathrm{Ru}$ is different for PLR, NLR, and SLR. Panaccione et al. ${ }^{31}$ suggested that with the increase of $U$ i.e., electron correlation the intensity of the screened peak decreases. Similar results have been

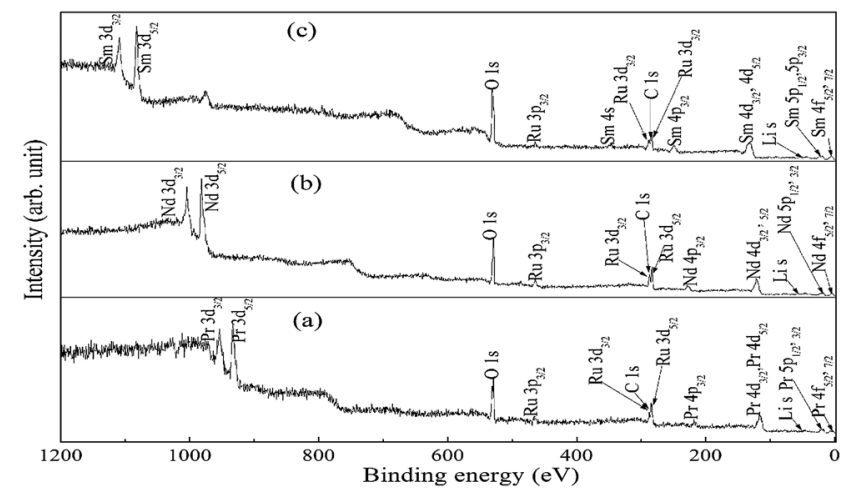

Fig. 6 XP spectra of (a) PLR, (b) NLR and (c) SLR. 


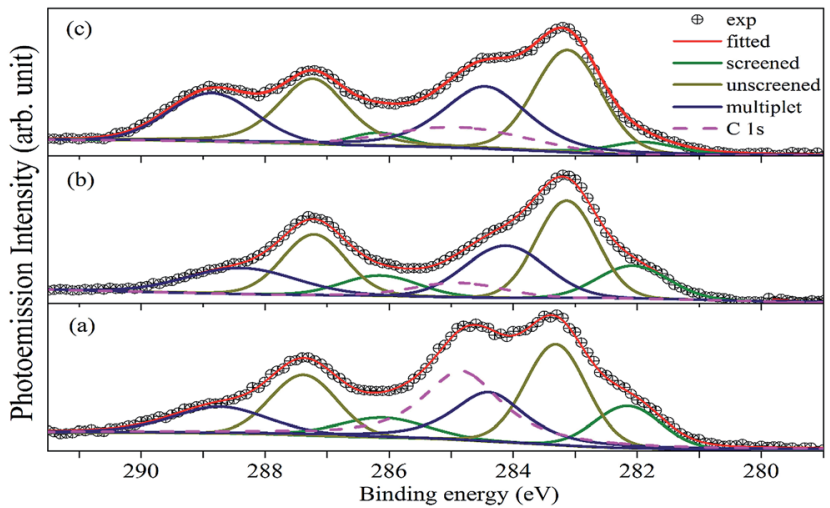

Fig. 7 Fitted Ru(3d)/C(1s) core-level spectra for (a) PLR, (b) NLR and (c) SLR. Symbols are the experimental data, solid green lines, dark yellow solid lines and blue solid lines represent the screened, unscreened and the multiplet peaks. The solid red lines are the profile of the sum of the peaks. The pink dashed line represents the $C$ 1s peaks.

obtained for the Ru 3d evolution as we move from PLR to SLR. The $U$ value is in the order of $\mathrm{Sm}>\mathrm{Nd}>\operatorname{Pr}$ (Table $\mathrm{S} 1 \dagger$ ), so accordingly the screened peak intensity is in the order of $\mathrm{Pr}>\mathrm{Nd}$ $>$ Sm (Table 1).

The core-level XP spectra of Pr 3d, Nd 3d and Sm 3d of the investigated compounds are shown in Fig. 8. Each of the spectra is split into two components due to the spin-orbit interaction having a lower $J(=l-s)$ value component in the higher energy side and a higher $J(=l+s)$ component in the lower energy side. The high intensity peaks at 932.93 and $953.47 \mathrm{eV}$ in PLR, 981.6 and $1004.1 \mathrm{eV}$ in NLR and $1082 \mathrm{eV}$ and $1109 \mathrm{eV}$ in SLR correspond to the $A 3 d_{5 / 2}$ and $A 3 d_{3 / 2}$ levels. This splitting is equal to 20.5, 22.5 and $27 \mathrm{eV}$ for $\mathrm{Pr}, \mathrm{Nd}$, and Sm respectively. In addition to this, the Pr $3 \mathrm{~d}$ and $\mathrm{Nd} 3 \mathrm{~d}$ profiles show interesting features and are fitted to locate the positions of the main and the screened peaks. At the low-binding-energy side of the main lines of the $3 d_{5 / 2}$ and $3 d_{3 / 2}$, the peaks at 928.43 and $948.77 \mathrm{eV}$ for PLR and 977.75 and $999.69 \mathrm{eV}$ for NLR are observed which is known to account for the screened $\operatorname{Pr} 3 \mathrm{~d}^{9} 4 \mathrm{f}^{3}$ and Nd $3 \mathrm{~d}^{9} 4 \mathrm{f}^{4}$ final states. $^{39,40}$ It is observed from Fig. 8 that the intensity of the screened peaks is less than that of the $3 \mathrm{~d}$ main peaks. The origin of these peaks can be attributed to life time effects and the exchange interaction between the $3 \mathrm{~d}$ hole and the partially filled $4 \mathrm{f}$ shell of $\mathrm{Pr}^{3+}$ and $\mathrm{Nd}^{3+}$ ion. ${ }^{41-43}$ It has been observed that the screened peaks are well separated from the main $3 \mathrm{~d}$ peaks in the case of PLR and NLR, however, no significant peaks are

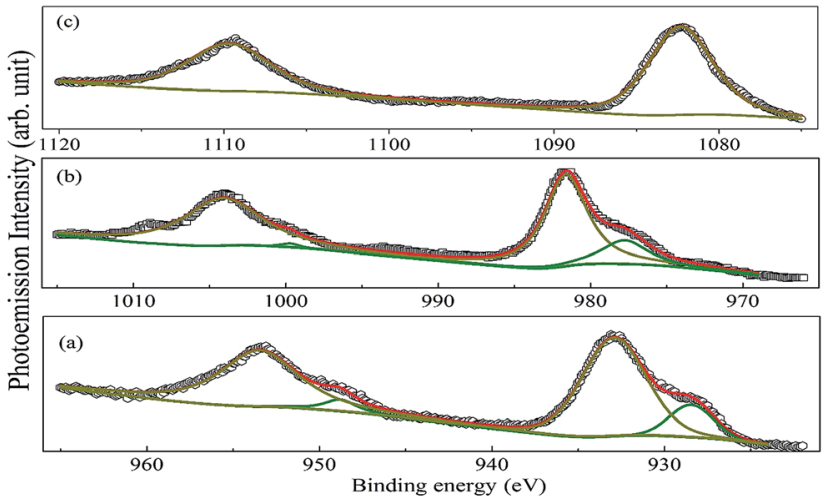

Fig. 8 XP spectra of Pr 3d, Nd 3d and Sm 3d for (a) PLR, (b) NLR and (c) SLR. Symbols are the experimental data, solid green lines and dark yellow solid lines represent the screened and unscreened peaks. The solid red lines are the profile of the sum of the peaks.

observed in SLR. Hillerbrecht and Fuggle ${ }^{41}$ found similar results for $\mathrm{LnPd}_{3}(\mathrm{Ln}=\mathrm{La}, \mathrm{Ce}, \mathrm{Pr}, \mathrm{Nd}$, and $\mathrm{Sm})$ compounds. According to Hillerbrecht et al. the observation of these screened peaks is due to the hybridization of $4 \mathrm{f}$ levels with the extended $\mathrm{CB}$ states ${ }^{41,44}$ in $\operatorname{LnPd}_{3}(\mathrm{Ln}=\mathrm{La}, \mathrm{Ce}, \mathrm{Pr}, \mathrm{Nd}$, and Sm). Further, based on the XP spectroscopy of these compounds they have concluded that the hybridization in $\operatorname{LnPd}_{3}$ decreases in the order $\mathrm{La}>\mathrm{Ce}>\mathrm{Pr}>\mathrm{Nd}>\mathrm{Sm}$. So, this may be the reason that no screened peaks have been observed for SLR.

In order to verify the electronic structure calculations experimentally, we have performed the VB-XP spectroscopy measurements of these compounds. The VB-XP spectrum of PLR, NLR and SLR are shown in Fig. 9(a)-(c) respectively (solid red lines). We have generated the VB spectrum of PLR, NLR and SLR using the calculated DOS in a standard way by adding the partial DOS of Pr 4f, Nd 4f, Sm 4f, Li p, Ru 4d and O 2p (multiplied with their respective photo-emission cross-section for $1486.6 \mathrm{eV}$ ) weighted by the atomic cross sections for photon scattering:.$^{45}$ The added DOS is convoluted with a Lorentzian of $0.55 \mathrm{eV}, 0.86 \mathrm{eV}$ and $0.9 \mathrm{eV}$ FWHM for PLR, NLR and SLR respectively. The calculated VB-DOS spectrum is compared with the experimental XP VB spectra, as shown in Fig. 9. The VB spectra of these materials from the Fermi level $\left(E_{\mathrm{F}}\right)$ to $\sim 2.5 \mathrm{eV}$ below it mainly consists of $\mathrm{Ru} 4 \mathrm{~d}$ states and the contribution of $\mathrm{O} 2 \mathrm{p}$ states lies from below $\sim 2.5 \mathrm{eV}$ to $\sim 12 \mathrm{eV}$ below $E_{\mathrm{F}}$. In order to align the major peak obtained theoretically with the experimental XP spectra, the theoretical DOS has been shifted. This

Table 1 Experimentally determined binding energies and the fit parameters for $\mathrm{A}_{2} \mathrm{LiRuO}_{6}$

\begin{tabular}{|c|c|c|c|c|c|c|c|c|c|c|}
\hline Material & Peak & $\mathrm{BE}(\mathrm{eV})$ & FWHM (eV) & $\begin{array}{l}\text { Doublet } \\
\text { separation }(\mathrm{eV})\end{array}$ & $\mathrm{BE}(\mathrm{eV})$ & FWHM (eV) & $\begin{array}{l}\text { Doublet } \\
\text { separation }(\mathrm{eV})\end{array}$ & $\mathrm{BE}(\mathrm{eV})$ & $\begin{array}{l}\text { FWHM } \\
(\mathrm{eV})\end{array}$ & $\begin{array}{l}\text { Doublet } \\
\text { separation }(\mathrm{eV})\end{array}$ \\
\hline PLR & $\mathrm{Ru} 3 \mathrm{~d}_{5 / 2}$ & 282.1 & 1.251 & 4 & 283.31 & 1.152 & 4.066 & 284.39 & 1.357 & 4.332 \\
\hline \multirow[t]{2}{*}{ NLR } & $\mathrm{Ru} 3 \mathrm{~d}_{5 / 2}$ & 282.067 & 1.337 & 4.07 & 283.14 & 1.13 & 4.07 & 284.122 & 1.538 & 4.26 \\
\hline & $\mathrm{Ru} 3 \mathrm{~d}_{3 / 2}$ & 286.141 & 1.440 & & 287.21 & 1.19 & & 288.379 & 2.064 & \\
\hline SLR & $\mathrm{Ru} 3 \mathrm{~d}_{5 / 2}$ & 282.2 & 1.38 & 4.1 & 283.227 & 1.229 & 4.04 & 284.533 & 1.438 & 4.33 \\
\hline
\end{tabular}



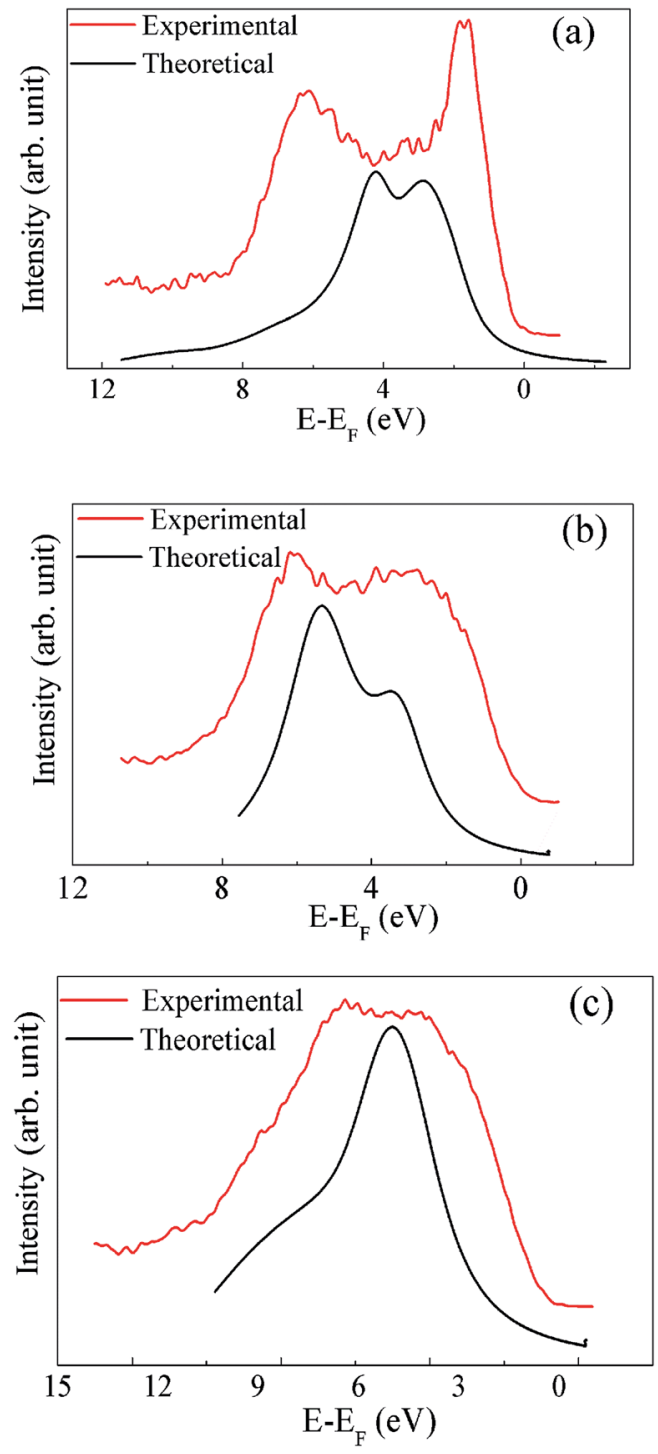

Fig. 9 The XP spectrum in the valence band region are compared with the theoretically calculated spectrum for (a) PLR, (b) NLR and (c) SLR.

shift has been performed because, in the experiment, the energy zero is taken as the "middle of the gap", while, in theory, it is taken at the top of the VB. ${ }^{46}$ Further, according to Ong et al. ${ }^{46}$ there may be a "homogeneous charging" problem for insulators which can shift the entire spectrum rigidly without any distortion of broadening and without changing the relative positions of the various spectroscopic features. The calculated DOS for all of the studied materials show sharper peaks than the experimental spectrum, which may be due to the fact that in the DFT calculations the lifetime broadening is not taken into account.

\subsection{Dielectric tensor}

The contribution of the electronic and the ionic dielectric constant has been calculated from the Born effective charges (BECs) of the constituent atoms of the system. The calculated electronic and ionic parts of static dielectric tensor for $\mathrm{A}_{2} \mathrm{LiRuO}_{6}$ are given in section S3 in ESI. $\dagger$ The calculated dielectric tensors for all these materials are anisotropic reflecting the lowsymmetric structure. The average value of the electronic and the ionic dielectric constant is found to be 6.86 and 19 for PLR, 5.97 and 23.2 for NLR, 5.73 and 17.1 for SLR respectively. The obtained results clearly indicate that the dielectric constant value is dominated by the ionic component. Thus, the total static dielectric constant value obtained by summing up the mode contributions and the electronic dielectric constant are 25.86, 29.2 and 22.8 for PLR, NLR and SLR respectively.

\section{Conclusion}

The double perovskite $\mathrm{A}_{2} \mathrm{LiRuO}_{6}(\mathrm{~A}=\mathrm{Pr}, \mathrm{Nd}, \mathrm{Sm})$ compounds have been synthesized by the solid-state reaction method. The Rietveld refinement of the XRD data indicates that the roomtemperature crystal structure of $\mathrm{A}_{2} \mathrm{LiRuO}_{6}$ is monoclinic with the space group $P 2_{1} / n$, which contains an ordered array of alternate $\mathrm{LiO}_{6}$ and $\mathrm{RuO}_{6}$ octahedra. The calculated bond distances and bond angles show good agreement with the experimental data. The optical properties of these materials have been predicted by the first-principles calculations and all these crystals exhibit positive uniaxial anisotropy. The observed anisotropy in these materials primarily arises from the asymmetrically oriented $\mathrm{Ru}-\mathrm{O}$ bonds in $\mathrm{RuO}_{6}$ octahedra in the unit cell. The interband contributions to $\varepsilon_{2}(\omega)$ of these materials are explained using the band structures. The evolution of $\mathrm{Ru} 3 \mathrm{~d}$ spectra in these materials suggests that the electron-correlation strength is minimum in PLR and maximum in SLR among these materials. The VB-XP spectra are obtained and compared with the calculated DOS spectra in the VB region. It has been established that the $\mathrm{O} 2 \mathrm{p}$ states hybridize with the $\mathrm{Ru} d$ states and occupy the VB maximum. The calculated dielectric constant values are dominated by the ionic component. The orientationally averaged dielectric constant has been calculated and is found to be the highest for NLR among these materials which suggests that NLR may be used as a high dielectric constant material for microwave applications.

\section{Conflicts of interest}

There are no conflicts to declare.

\section{Acknowledgements}

Anup Pradhan Sakhya and Md. Sariful Sheikh acknowledges Department of Science and Technology for providing the financial support through Inspire Fellowship. Alo Dutta thanks the Department of Science and Technology of India for providing the financial support through DST Fast Track Project under grant no. SB/FTP/PS-175/2013. D. P. Rai acknowledges a Start-Up Grant from UGCBSR, FRPS, Lett. No. F.30-52/2014.

\section{References}

1 R. H. Mitchell, Perovskites: Modern and Ancient, Almaz Press, Ont., Canada, 2002. 
2 C. Martin, A. Maignan, M. Hervieu, B. Raveau and J. Hejtmanek, Eur. Phys. J. B, 2000, 16, 469-474.

3 B. Raveau, A. Maignan, C. Martin, R. Mahendiran and M. Hervieu, J. Solid State Chem., 2000, 151, 330.

4 Y. Maeno, H. Hashimoto, K. Yoshida, S. Nishizaki, T. Fujita, J. G. Bednorz and F. Lichtenberg, Nature, 1994, 372, 532-534.

5 K. M. Glassford and J. R. Chelikowsky, Phys. Rev. B: Condens. Matter Mater. Phys., 1993, 47, 1732-1741.

6 T. Yamamoto, R. Kanno, Y. Takeda, O. Yamamoto, Y. Kawamoto and M. J. Takano, Solid State Chem., 1994, 109, 372-383.

7 Y. Maeno, H. Hashimoto, K. Yoshida, S. Nishizaki, T. Fujita, J. G. Bednorz and F. Lichtenberg, Nature, 1994, 372, 532-534. 8 P. Khalifah, R. Osborn, Q. Huang, H. W. Zandbergen, R. Jin, Y. Liu, D. Mandrus and R. J. Cava, Science, 2002, 297, 22372240.

9 P. Khalifah, K. D. Nelson, R. Jin, Z. Q. Mao, Y. Liu, Q. Huang, X. P. A. Gao, A. P. Ramirez and R. J. Cava, Nature, 2001, 411, 669-671.

10 L. Klein, J. S. Dodge, C. H. Ahn, J. W. Reiner, L. Mieville, T. H. Geballe, M. R. Beasley and A. Kapitulnik, J. Phys.: Condens. Matter, 1996, 8, 10111-10126.

11 H. D. Kim, H. J. Noh, K. H. Kim and S. J. Oh, Phys. Rev. Lett., 2004, 93, 126404.

12 S. J. Makowski, J. A. Rodgers, P. F. Henry, J. P. Attfield and J. W. G. Bos, Chem. Mater., 2009, 21, 264-272.

13 S. Ghosh, W. H. Wang, F. M. Mendoza, R. C. Myers, X. Li, N. Samarth, A. C. Gossard and D. D. Awschalom, Nat. Mater., 2006, 5, 261-264.

14 K. Aoki, H. T. Miyazaki, H. Hirayama, K. Inoshita, T. Baba, K. Sakoda, N. Shinya and Y. Aoyagi, Nat. Mater., 2003, 2, 117-121.

15 S. J. Goncher, N. E. Sveum, D. T. Moore, N. D. Bartlett and D. M. Neumarka, J. Chem. Phys., 2006, 125, 224304.

16 H. Zhang, M. Zhang, S. Pan, Z. Yang, Z. Wang, Q. Bian, X. Hou, H. Yu, F. Zhang, K. Wu, F. Yang, Q. Peng, Z. Xu, K. B. Chang and K. R. Poeppelmeier, Cryst. Growth Des., 2015, 15, 523-529.

17 Q. Bian, Z. Yang, L. Dong, S. Pan, H. Zhang, H. Wu, H. Yu, W. Zhao and Q. Jing, J. Phys. Chem. C, 2014, 118, 2565125657.

18 A. P. Sakhya, D. P. Rai, Md. S. Sheikh, A. Dutta, R. K. Thapa and T. P. Sinha, RSC Adv., 2016, 6, 59988-59997.

19 A. H. Reshak, H. Kamarudin, I. V. Kityk and S. Auluck, J. Phys. Chem. B, 2012, 116, 13338-13343.

20 J. R. Carvaja, Physica B, 1993, 192, 55-69.

21 P. E. Blöchl, Phys. Rev. B: Condens. Matter Mater. Phys., 1994, 50, 17953-17979.

22 G. Kresse and D. Joubert, Phys. Rev. B: Condens. Matter Mater. Phys., 1999, 59, 1758-1775.

23 G. Kresse and J. Furthmuller, Phys. Rev. B: Condens. Matter Mater. Phys., 1996, 54, 11169-11186.
24 P. Blaha, K. Schwarz, G. K. H. Madsen, D. Kvasnicka, J. Luitz and K. Schwarz, An augmented plane wave plus local orbitals program for calculating crystal properties, Wien $2 K$ User's Guide, Techn. Universitat Wien, Wien, 2008.

25 P. M. Woodward, Acta Crystallogr., Sect. B: Struct. Sci., 1997, 53, 44-66.

26 A. H. Reshak, Ph.D. thesis, Indian Institute of Technology Rookee, India, 2005.

27 A. H. Reshak, RSC Adv., 2016, 6, 51675-51682.

28 S. Cabuk, Philos. Mag., 2016, 96, 190-207.

29 Y. Noguchi, M. Soga, M. Takahashi and M. Miyayama, Jpn. J. Appl. Phys., 2005, 44, 6998-7002.

30 A. Roy, R. Prasad, S. Auluck and A. Garg, J. Appl. Phys., 2014, 115, 133509-133516.

31 G. Panaccione, U. Manju, F. Offi, E. Annese, I. Vobornik, P. Torelli, Z. H. Zhu, M. A. Hossain, L. Simonelli, A. Fondacaro, P. Lacovig, A. Guarino, Y. Yoshida, G. A. Sawatzky and A. Damascelli, New J. Phys., 2011, 13, 053059.

32 K. Maiti, Phys. Rev. B: Condens. Matter Mater. Phys., 2006, 73, 235110.

33 K. Maiti, Phys. Rev. B: Condens. Matter Mater. Phys., 2008, 77, 212407.

34 P. A. Cox, R. G. Egdellt, J. B. Goodenough, A. Hamnett and C. C. Naishj, J. Phys. C: Solid State Phys., 1983, 16, 6221.

35 H.-D. Kim, H.-J. Noh, K. H. Kim and S.-J. Oh, Phys. Rev. Lett., 2004, 93, 126404.

36 R. W. M. Kwok, see http://www.phy.cuhk.edu.hk/ surface/ XPSPEAK for XPS Peak Fitting Program Version 4.1, 2000.

37 D. A. Shirley, Phys. Rev. B: Condens. Matter Mater. Phys., 1972, 5, 4709-4714.

38 J. F. Moulder, W. F. Strickle, P. E. Sobol and K. D. Bomben, Handbook of X-ray Photoelectron Spectroscopy, Perkin-Elmer Corporation: Physical Electronics Division, Eden Prairie, MN, 1992.

39 J. C. Fuggle, F. U. Hillebrecht, Z. Żołnierek, R. Lässer, C. H. Freiburg, O. Gunnarsson and K. Schönhammer, Phys. Rev. B: Condens. Matter Mater. Phys., 1983, 27, 7330-7341.

40 A. Szytuła, B. Penc and A. Jezierski, Mater. Sci.-Pol., 2008, 26(3), 759-765.

41 F. U. Hillerbrecht and J. C. Fuggle, Phys. Rev. B: Condens. Matter Mater. Phys., 1982, 25(6), 3550-3556.

42 J. C. Fuggle and S. F. Alvarado, Phys. Rev. A, 1980, 22(4), 1615-1624.

43 J. C. Fuggle, M. Campagna, Z. Zolnierek, R. Lasser and A. Platau, Phys. Rev. Lett., 1980, 45(19), 1597-1600.

44 B. V. Kumar, R. Velchuri, G. Prasad, B. Sreedhar, K. Ravikumar and M. Vithal, Ceram. Int., 2010, 36, 13471355.

45 J. J. Yeh and I. Lindau, At. Data Nucl. Data Tables, 1985, 32, 1155.

46 K. P. Ong, P. Blaha and P. Wu, Phys. Rev. B: Condens. Matter Mater. Phys., 2008, 77, 073102-073104. 